\title{
ФОТОТРАНСФОРМАЦИЯ НЕОДНОРОДНО УШИРЕННОЙ ПОЛОСЫ ПОГЛОЩЕНИЯ ПОД ДЕИСТВИЕМ «БЕЛОГО» ИЗЛУЧЕНИЯ
}

\author{
(Представил К. К. Ребане)
}

\section{1. Введение}

В экспериментах по фотовыжиганию спектральных провалов ФСП [ $\left.{ }^{1}\right]$ широкополосное (по сравнению с неоднородной полосой) облучение часто применяется для восстановления исходного спектрального распределения выжженного образца, а также для исследования механизмов выжигания $\left[{ }^{2,3}\right]$. При этом наблюдаемые изменения (уменьшение интегральной оптической плотности, трансформация спектра) обычно интерпретируются как изменение соотношения относительных концентраций химически (и спектрально) неэквивалентных исходной примеси и фотопродукта.

В случае механизма выжигания, связанного не с химическим преобразованием самой молекулы, а с некоторой перестройкой ее ближайшего окружения (т. н. нефотохимическое выжигание) $\left[{ }^{4,5}\right]$, а также, например, при внутримолекулярной фототаутомеризации, наблюдаемой в безметальных симметричных порфиринах $\left[{ }^{6}\right]$ и эквивалентной повороту молекулы как целого, фотопродукт спектрально перераспределяется по исходной неоднородной полосе. Однако в подобном случае статистической спектральной идентичности фотопродукта и исходной молекулы существует ряд физических факторов, которые могут привести к трансформации спектра. Они рассматриваются в настоящем сообщении.

\section{2. Воздействие «белого» света на оптически толстый объект}

Здесь и далее термин оптически толстый объект применяется для описания образцов, в которых ослабление распространяющегося света вследствие поглощения не является пренебрежимо малым и требует учета. Если поглощение в таком образце спектрально неравномерное, то свет, распространяясь в нем, меняет свой спектральный состав. В частности, падающий «белый» свет, распространяясь в оптически толстом образце, перестает быть таковым. В фотовыжигаемой спектрально неоднородной системе излучение, имеющее спектр, отличный от «белого», вызывает, в свою очередь, перераспределение центров по неоднородной полосе и, следовательно, трансформацию этой полосы. 
Діля нахождения функции неоднородного распределения (ФНР) центров в трансформированной полосе поглощения воспользуемся моделью ([ $\left.{ }^{7}\right]$, п. 3), в которой центры могут находиться в одной из двух конформаций (которым соответствуют различные частоты бесфононной линии (БФЛ) перехода) и распределение центров по частотам считается независимым. ФНР для момента насыщения перераспределения центров $\tilde{\varrho}(\omega)$ выражается через исходную двумерную ФНР $\varrho_{0}\left(\omega_{1}, \omega_{2}\right)=\varrho_{0}\left(\omega_{1}\right) \varrho_{0}\left(\omega_{2}\right)$ и долю центров, имеющих БФЛ на частотах $\omega_{1}$ и $\omega_{2}$ и находящихся в данный момент в положении с частотой $\omega_{1}\left(N_{12}\right)$

$$
\tilde{\varrho}\left(\omega_{1}\right)=2 \varrho_{0}\left(\omega_{1}\right) \int_{-\infty}^{\infty} \varrho_{0}\left(\omega_{2}\right) N_{12}\left(\omega_{1}, \omega_{2}\right) d \omega_{2} .
$$

$\Phi Н Р$ $\varrho(\omega)$ предполагаем имеющей гауссовый контур с дисперсией $\sigma$. Функция $N_{12}\left(\omega_{1}, \omega_{2}\right)$ зависит от спектрального распределения интенсивности излучения, достигшего рассматриваемой глубины $x(I(\omega, x))$

$$
\begin{gathered}
N_{12}\left(\omega_{1}, \omega_{2}\right)=\int_{-\infty}^{\infty} d \omega^{\prime} I\left(\omega^{\prime}, x\right) \\
x\left(\omega^{\prime}-\omega_{2}\right) / \int_{-\infty}^{\infty} d \omega^{\prime \prime} I\left(\omega^{\prime \prime}, x\right)\left[\varkappa\left(\omega^{\prime \prime}-\omega_{2}\right)+\right. \\
\left.+\varkappa\left(\omega^{\prime \prime}-\omega_{1}\right)\right],
\end{gathered}
$$

где $x\left(\omega^{\prime}-\omega_{i}\right), i=1,2-$ однородный спектр поглощения, имеющий максимум БФЛ на частоте $\omega_{i}$.

Прохождение света через объект и его поглощение в нем описывается уравнением

$$
\partial I(v, x) / \partial x=-I(v, x) \int_{-\infty}^{\infty} d \omega \tilde{\varrho}(\omega) x(v-\omega) .
$$

Однородный спектр поглощения $x(v-\omega)$ моделируем суммой БФЛ (в виде $\delta$-функции) и фононного крыла (ФК) в виде гауссиана, имеющего дисперсию $\sigma_{\Phi \mathrm{K}}=0,5 \sigma$ и положение максимума, сдвинутое относительно БФЛ на собственную полную ширину на половине высоты (т. е. $\left.2(2 \ln 2)^{1 / 2} \sigma_{\Phi K}\right)$

$$
\begin{gathered}
x(v-\omega)=\alpha \delta(v-\omega)+\left((1-\alpha) /\left(\sqrt{2 \pi \sigma_{\Phi K}}\right)\right) \times \\
\times \exp \left\{-\left(v-\omega-2(2 \ln 2)^{1 / 2} \sigma_{\Phi K}\right)^{2} / 2 \sigma_{\Phi \mathrm{K}}^{2}\right\} .
\end{gathered}
$$

Здесь параметр $\alpha$ имеет смысл фактора Дебая-Валлера.

На рис. 1 изображена зависимость оптической толщины в максимуме полосы поглощения (в состоянии динамического равновесия перераспределения центров по полосе) $D_{\infty}$ от оптической плотности в максимуме полосы в начальный момент времени $D_{0}$. Кривые, полученные путем численного решения уравнения (3), соответствуют различным значениям фактора Дебая-Валлера $\alpha$.

При прохождении света через оптически плотную среду его интенсивность на краях полосы поглощения уменьшается медленее, чем в максимуме полосы, что вызывает некоторое перераспределение центров к максимуму полосы и увеличение оптической плотности в нем. Изображенные на рис. 1 кривые иллюстрируют это увеличение. Как видно из рис. 1, наличие ФК существенно уменьшает эффект нарастания оптической плотности. Кроме изменения формы неоднородной полосы происходит ее сдвиг, вызванный наличием в поглощении $Ф \mathrm{~K}$, 


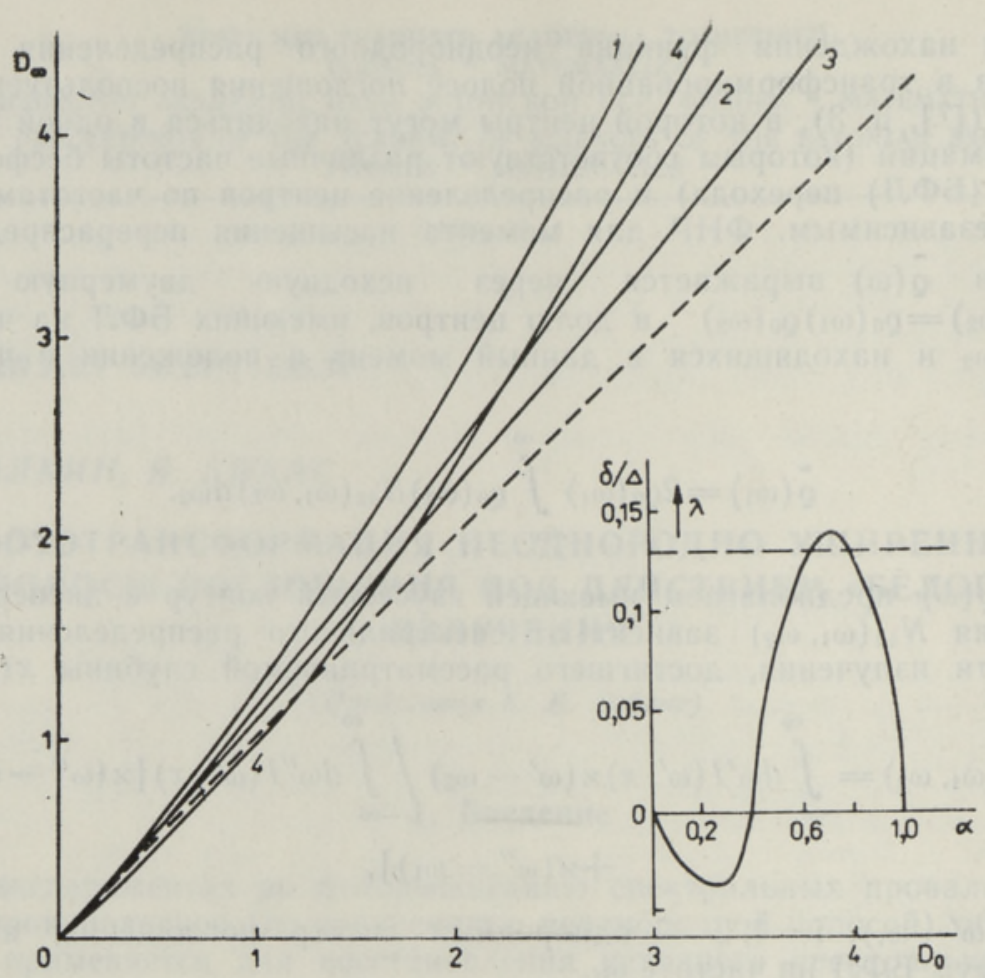

Рис. 1. Зависимость оптической толщины в максимуме полосы поглощения (при насыщении перераспределения центров по полосе) $D_{\infty}$ от таковой в начальный момент времени $D_{0}$. Штриховая линия соответствует $D_{\infty}=D_{0} .1,2,3-$ фактор ДебаяВаллера $\boldsymbol{\alpha}=1,0,0,8,0,5$ соответственно. Кривая 4 иллюстрирует уменьшение нарастания плотности при учете пространственной переориентации примесей (по сравнению с кривой 1) при $\alpha=1,0$.

На вставке изображен относительный сдвиг максимума полосы поглощения (отношение сдвига $s$ к полной ширине на половине высоты $\Delta$ исходной полосы) при начальной оптической плотности 4,0. Положительное направление оси соответствует сдвигу в сторону больших длин волн. Штриховая линия - экспериментальное значение $s / \Delta$, полученное для $D_{0}=4,30$.

причем направление сдвига зависит от соотношения интегральных интенсивностей БФЛ и крыла, т. е. фактора $\alpha$. Вставка на рис. 1 демонстрирует относительный сдвиг максимума полосы поглощения при начальной оптической плотности 4 (положительное направление оси соответствует увеличению длины волны). Разумеется, эта зависимость имеет приведенный вид при упомянутых значениях параметров $\sigma_{\Phi \mathrm{K}}$ и сдвига ФК относительно БФЛ.

\section{3. Пространственная переориентация примеси}

Рассмотренная в разделе 2 модель, по-видимому, довольно реалистично описывает случай структурной перестройки ближайшего окружения молекулы. Если же процесс выжигания связан с поворотом молекулы (или ее фрагментов) на большие углы, то следует учесть и уменьшение проекции дипольного момента перехода на плоскость падающего света, которое происходит из-за тенденции перехода центров 
в состояния наименьшего поглощения при экспозиции объекта «белым» неполяризованным светом. В предположении отсутствия корреляции между возможными для данной примеси пространственными ориентациями и частотами БФЛ в этих ориентациях в оптически тонком образце интенсивность полосы поглощения уменьшается, причем во всех спектральных точках в равной степени.

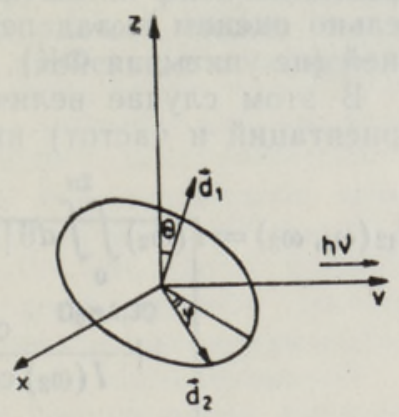

Рис. 2. Две возможные орнентации дипольного момента перехода, угол между которыми составляет $90^{\circ}$. Примесный центр помещен в начало координат, свет распространяется вдоль оси $y$.

Оценим происходящее уменьшение в оптически тонком объекте для системы центров, имеющих два возможных положения дипольного момента перехода, угол между которыми составляет $90^{\circ}$ (модель, соответствующая внедренным в матрицу молекулам безметальных порфиринов $\left[{ }^{6}\right]$ ) (рис. 2). Предположим, что примесный центр помещен в начало координат, а плоскость $z x$ - плоскость падающего неполяризованного света. Пусть одно из возможных положений вектора дипольного момента $\left(\mathbf{d}_{1}\right)$ находится в плоскости $z y$ и составляет угол $\theta$ c осью $z$. Тогда второе возможное положение $\left(\mathbf{d}_{2}\right)$ находится в плоскости, перпендикулярной $\mathbf{d}_{1}$, и составляет некоторый угол $\varphi$ с линией пересечения этой плоскости и плоскости $z y$ (см. рис. 2). Среднее поглощение всех примесей, для которых возможна ориентация дипольного момента вдоль $\mathbf{d}_{1}$ и $\mathbf{d}_{2}$ и которые пребывают в рассматриваемый момент в одном из этих состояний $\mathbf{d}_{i}(i=1,2)$, пропорционально произведению вероятности пребывания центра в этом положении $P_{i}$ и квадрату проекции вектора дипольного момента на плоскость $z x\left(d_{i}{ }^{2}\right)$. Квадрат проекции вектора $\mathbf{d}_{1}$ пропорционален $\cos ^{2} \theta$, а вектора $\mathbf{d}_{2}-\left(1-\cos ^{2} \theta \cos ^{2} \varphi\right)$. Начальные вероятности равны 0,5 ; после того как в процессе перераспределения примесей наступит динамическое равновесие, $P_{1}=d_{2}^{2} /\left(d_{1}^{2}+d_{2}^{2}\right)$ и $P_{2}=d_{1}^{2} /\left(d_{1}^{2}+d_{2}^{2}\right)$. Суммарное поглощение системы переориентирующихся центров пропорционально интегралу от суммы $P_{1} d_{1}^{2}+P_{2} d_{2}^{2}$ по всем возможным углам $\theta$ и $\varphi$. (Интегрировать по третьему пространственному углу, например, отклонению вектора $\mathbf{d}_{1}$ от плоскости $z y$ не нужно, так как интеграл вносит лишь постоянный коэффициент, пропорциональный $|\cos \theta|$ из-за общей симметрии всей схемы относительно оси распространения света $y$.) Интеграл для начального состояния системы $J_{0}$ равен:

$$
J_{0}=0,5 \int_{0}^{2 \pi}|\cos \theta| d \theta \int_{0}^{2 \pi} d \varphi\left(\cos ^{2} \theta+1-\cos ^{2} \theta \cos ^{2} \varphi\right)=16 \pi / 3 .
$$

Интеграл, соответствующий системе, в которой процесс переориентации достиг равновесия, равен:

$$
\begin{gathered}
J_{\infty}=2 \int_{0}^{2 \pi}|\cos \theta| d \theta \cos ^{2} \theta \int_{0}^{2 \pi} d \varphi\left(1-\cos ^{2} \varphi \cos ^{2} \theta\right) / \\
/\left(\cos ^{2} \theta+1-\cos ^{2} \varphi \cos ^{2} \theta\right) \approx 13,77 .
\end{gathered}
$$


Таким образом, при облучении системы переориентирующихся на $90^{\circ}$ центров «белым» светом оптическая плотность в неоднородно уширенной полосе составляет $J_{\infty} / J_{0} \approx 0,82$ от исходной величины.

Эта оценка сделана для тонкого объекта при пренебрежении наличием фононных крыльев. Описание модели, учитывающей совместно нарастание оптической плотности в толстом объекте и наличие фононного крыла, связано с вычислительными трудностями, поэтому приблизительно оценим вклад переориентаций, считая однородный спектр $\delta$-линией (не учитывая ФК).

В этом случае величину $N_{12}\left(\omega_{1}, \omega_{2}\right)$ (при отсутствии корреляции ориентаций и частот) вместо (2) опишет следующее выражение:

$$
\begin{gathered}
N_{12}\left(\omega_{1}, \omega_{2}\right)=I\left(\omega_{2}\right) \int_{0}^{2 \pi} \int_{0}^{2 \pi} d \theta|\cos \theta| d \varphi\left\{\frac{\cos ^{2} \theta\left(1-\cos ^{2} \varphi \cos ^{2} \theta\right)}{I\left(\omega_{1}\right) \cos ^{2} \theta+I\left(\omega_{2}\right)\left(1-\cos ^{2} \varphi \cos ^{2} \theta\right)}+\right. \\
\left.+\frac{\cos ^{2} \theta\left(1-\cos ^{2} \varphi \cos ^{2} \theta\right)}{I\left(\omega_{2}\right) \cos ^{2} \theta+I\left(\omega_{1}\right)\left(1-\cos ^{2} \theta \cos ^{2} \varphi\right)}\right\} .
\end{gathered}
$$

Выразив через (7) функцию $\tilde{\varrho}(\varphi)$ (1) и подставив ее в (3), получим уравнение, описывающее распространение света для этой модели. Кривая 4 на рис. 1 демонстрирует численное решение этого уравнения. Видно, что учет переориентаций примесей приводит к уменьшению эффекта нарастания плотности в максимуме полосы (в сравнении с кривой 1). Можно ожидать, что при совместном учете переориентаций и сдвига полосы, связанного с наличием фононного крыла, этот эффект будет еще меньше; а превышение оптической плотности по сравнению с начальной величиной наблюдается при значительно большей начальной оптической плотности, чем в случае, когда сдвиг полосы не учитывается.

\section{4. Эксперимент}

Әксперимент по воздействию «белого» света проводился на 0 -0-полосе (положение максимума около 619 нм) октаэтилпорфина (ОЭП) в матрице полистирола. Механизм фототрансформации в ОЭП хорошо известен и заключается в повороте на $90^{\circ}$ пары центральных протонов внутри порфинового кольца $\left[{ }^{6}\right]$, что соответствует описываемому в данной работе случаю попадания выжженных молекул в исходную неоднородную полосу. В качестве источника «белого» света использовалась лампа накаливания ОИ-24 (рабочий ток 8,5 А) со светофильтрами КС-10, СЗС-26, ЖЗС-18, обеспечивающими постоянную интенсивность света в диапазоне 614-622 нм и спад интенсивности в «синюю» и «красную» стороны света от этого участка. Интегральная плотность излучения на поверхности объекта 55 мВт/см ${ }^{2}$ (около 200 мкВТ/( $\mathrm{cm}^{2} \cdot$ нм $)$ в районе 620 нм). Объекты, представляющие собой стопки разной толщины из полистирольных пленок, активированных ОЭП, экспонировались при температуре $4,2 \mathrm{~K}$ в течение 40 мин. До и после облучения снимался спектр пропускания ОЭП по стандартной методике (с помощью той же самой лампы накаливания, ослабляющих светофильтров, монохроматора ДФС-24). Интенсивность регистрируемого света выравнивалась при обоих измерениях по излучению с длиной волны 640 нм, поглощением света на которой пренебрегли.

На рис. 3 изображена полоса $0-0$-поглощения ОЭП до (1) и после (2) облучения «белеым» светом для четырех значений начальной оптической плотности. Отметим следующие происшедшие с полосой поглощения изменения. Оп்тическая плотность в максимуме полосы погло- 
щения при малой начальной плотности $\left(D_{0}=0,125\right)$ уменьшалась и составляла $0,85-0,9$ от исходной. Это изменение было меньше, чем полученная в разделе 3 оценка для оптически тонкого объекта $(0,82$ от исходной плотности). Возможной причиной такого отличия может быть отсутствие полной изотропности объекта, т. е. концентрации примесей разных ориентаций могут не быть одинаковыми. В таком случае в интегралы (5) и (6) входят соответствующие функции распределения примесей по ориентациям. Результатом такого расчета может стать другая оценка изменения оптической плотности, зависящая от конкретного вида функции распределения.

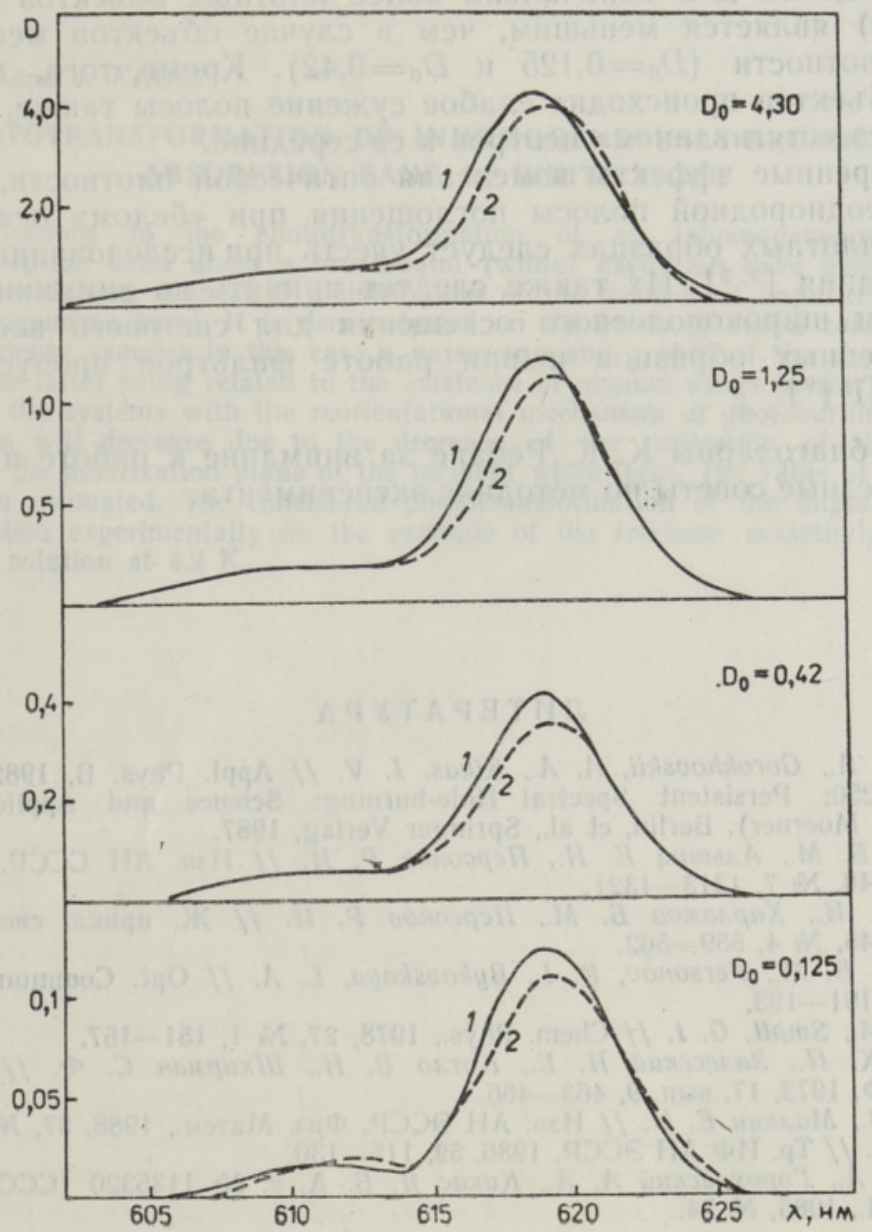

Рис. 3. Полоса 0-0-поглощения ОЭП до (1) и после (2) облучения «белым» светом для четырех значений начальной оптической плотности.

С увеличением начальной оптической плотности плотность в максимуме полосы поглощения изменяется еще слабее. При больших плотностях $\left(D_{0}=4,30\right)$ это изменение не превышает $5 \%$, однако все же не происходит и нарастания оптической плотности сверх начального уровня в максимуме полосы. По-видимому, это связано с наличием фононных крыльев, вызывающих сдвиг полосы в сторону больших длин 
волн. Величина этого сдвига очень мала для объекта с начальной плотностью 0,125 , а с увеличением начальной плотности растет, и для $D_{0}=4,30$ составляет $13 \%$ от полной ширины линии на половине высоты. Такой сдвиг соответствует теоретической оценке при значении фактора Дебая-Валлера $\sim 0,8$ (см. рис. 1), что для ОЭП при $4,2 \mathrm{~K}$ является разумной оценкой и согласуется с данными по ФСП в ОЭП (см. напр., $\left.\left[{ }^{8}\right]\right)$. Поскольку анализ совместного эффекта наличия асимметрии линии, связанной с фононными крыльями, и влияния переориентаций не проводился, трудно дать точную оценку, насколько должна измениться оптическая плотность в максимуме неоднородной полосы поглощения. Отметим, что процессы такого рода все же происходят, т. к. относительное изменение оптической плотности в максимуме полосы поглощения для изначально более плотных объектов $\left(D_{0}=1,25\right.$ и $\left.D_{0}=4,30\right)$ является меньшим, чем в случае объектов меньшей начальной плотности $\left(D_{0}=0,125\right.$ и $\left.D_{0}=0,42\right)$. Кроме того, для более плотных объектов происходит слабое сужение полосы также, вероятно, связанное со «стягиванием» центров к ее середине.

Рассмотренные эффекты изменения оптической плотности, сдвига и сужения неоднородной полосы поглощения при «белом» освещении в оптически плотных образцах следует учесть при исследовании механизмов выжигания $[2,3]$. Их также следует принять во внимание при использовании широкополосного освещения для светового восстановления выжженных образцов и при работе фильтров пропускания на основе ФСП $\left[{ }^{9}\right]$.

Авторы благодарны К. К. Ребане за внимание к работе и И. Сильдосу за полезные советы по методике эксперимента.

\section{ЛИТЕРАТ У РА}

1. Rebane, L. A., Gorokhovskii, A. A., Kikas. J. V. // Appl. Phys. B, 1982. 29, № 4, 235-250; Persistent Spectral Hole-burning: Science and Applications (ed. W. E. Moerner). Berlin, et al., Springer Verlag, 1987.

2. Харламов Б. М., Альщuц Е. Н., Персонов Р. Н. // Изв. АН СССР. Сер. физ., 1984,48, № $7,1313-1321$.

3. Альшиц Е. Н., Харламов Б. М., Персонов Р. И. // Ж. прикл. спектроскопии, 1986, 45, № 4, 559-562.

4. Kharlamov, B. M., Personov, R. I., Bykovskaya, L. A. // Opt. Commun., 1974, 12, № $1,191-193$.

5. Hayes, J. M., Small, G. J. // Chem. Phys., 1978, 27, № 1, 151-157.

6. Соловьев K. Н., Залесский $H$. Е., Котло В. Н., Шкирман С. Ф. // Письма в ЖЭТФ, 1973, 17, вып. 9, 463-466.

7. Кикас Я. В., Малкин Е. И. // Изв. АН ЭССР. Физ. Матем., 1988, 37, № 1, 47-51.

8. Кикас Я. В. // Тр. ИФ АН ЭССР, 1986, 59, 115-130.

9. Ребане Л. А., Гороховский А. А., Кикас Я. В. А. с. № 1135320 (СССР). Опубл. в Б. И., 1985 , № 34 .

\footnotetext{
Ннститут физики

Академии наук Эстонской ССР
}

Поступила в редакцию $19 /$ I 1988 


\section{MITTEHOMOGEENSELT LAIENENUD NEELDUMISRIBA FOTOTRANSFORMATSIOON «VALGE» VALGUSE TOIMEL}

On uuritud faktoreid, mis põhjustavad lisandi mittehomogeense neeldumisriba fototransformatgiooni kłiritamisel laịaribalise («valge») valgusega süsteemides, kus foto-* produkt jaotub spektraalselt ümber algse neeldumisriba piires. Mudelarvutuse alusel on näidatud, et optiliselt paksudes objektides leiab sellisel juhul aset neeldumisriba kitsenemine ja nihe, viimane on seotud foonontiibade olemasoluga homogeenses spektris. On hinnatud integraalse neeldumise kahanemist süsteemides, kus spektraalsälkamist põhjustab lisandi orientatsiooni muutus ja sellega kaasnev ülemineku dipoolmomendi projektsiooni vähenemine ergastava valguse polarisatsioonitasandile. Lisandispektri fototransformatsiooni on eksperimentaalselt uuritud oktaetüülprofiini tahke lahuse näitel polüstüroolis $(T=4,2 \mathrm{~K})$.

\section{Ye. MALKIN and J. KIKAS}

\section{PHOTOTRANSFORMATION OF INHOMOGENEOUSLY-BROADENED ABSORPTION BAND BY WHITE LIGHT}

Factors leading to the phototransformation of an inhomogeneously-broadened impurity absorption band under a broad-band (white) excitation have been studied for the systems, exhibiting a spectral redistribution of the burned-out impurities within the initial inhomogeneous band. It is demonstrated on the bases of model calculations that for optically dense samples in this case a narrowing and a shift of the absorption band take place, the latter being related to the existence of phonon-wings in the homogeneous spectrum. In the systems with the reorientational mechanism of photoburning the integral absorption will decrease due to the decrease of the projection of the transition moment onto the polarization plane of the incident white light. The value of the decrease has also been estimated. The considered phototransformation of the impurity spectrum has been studied experimentally on the example of the freebase octaethylporphin polystyrene solid solution at $4.2 \mathrm{~K}$. 